\title{
Impact of Plant Growth Regulators on Growth, Phenology and yield Characters of Hybrid Rice
}

\author{
Pawan Kumar Goutam $^{1 *}$, S.P. Kushwaha1, Dharmadew Chauhan², \\ Vikas Yadav ${ }^{1}$ and Subhash Kumar ${ }^{1}$
}

${ }^{1}$ Department of Crop Physiology, ${ }^{2}$ Department of Biochemistry, College of Agriculture, C.S. Azad University of Agriculture \& Technology, Kanpur -208 002, India

*Corresponding author

A B S T R A C T

Keywords

Foliar application, $\mathrm{GA}_{3}$, IAA, NAA, Triacontanol (TRIA), Cycocel, Growth parameters, Phenology, Nitrogen content in straw (\%), Yield, Hybrid rice

Article Info

Accepted:

07 December 2018 Available Online: 10 January 2019
The influence of foliar applied plant growth regulators on rice crop was conducted on hybrid rice crop during kharif season's 2015 and 2016. Application of five growth regulators each in two concentration viz., $\mathrm{GA}_{3}$ 20 and 40 ppm, IAA 25 and 50 ppm, NAA 10 and 20 ppm, Triacontanol 5 and $10 \mathrm{ppm}$, Cycocel 3000 and $4000 \mathrm{ppm}$ and one unsprayed control with foliar sprays at tillering and flowering stages for growth, yield parameters. The observation was recorded at different stages of crop. Higher concentration of IAA produce maximum dry biomass, increased the total leaf area plant $^{-1}$, total number of leaves at different crop growth stages, CCC delayed flowering and maturity, IAA produced higher nitrogen content in straw $(\%)$ and grain yield plant ${ }^{-1}(\mathrm{~g})$ over control $\left(\mathrm{T}_{1}\right)$.

\section{Introduction}

Rice is one of the staples food crop in India and most of country, its food provide energy, carbohydrate, proteins, vitamins consumed by majority of the population. World rice production 490.8 million metric tonnes and India rice production 109.7 million metric tonnes in year 2016-17 (USDA 2018). In India rice play key role it contributes also $46 \%$ of
Indian cereal production and also staple food for two third of the population. India has 433.88 lakh hectares in area and production 104.32 million tones in year 2015- 16 (DAC\& FW 2016-17). National Food Security Mission target of 3 million hectares, area under hybrid rice continue to range from 1.8 to 2.0 million hectares in the last few years. Hybrid rice plays a most important role for increase is yield in unit area. The yield advantage of 15- 
$20 \%$ over the best pure line varieties $\left(6.5 \mathrm{tha}^{-1}\right.$ as against $5.4 \mathrm{t} \mathrm{ha}^{-1}$ ) proved the key factor for wide adoption of the hybrid rice technology. Plant growth regulators play an essential role in regulating plant growth and development. Growth regulator is only needed in small amounts to determine the course of physiological processes, growth, differentiation, and development (Davies, 1995).

\section{Materials and Methods}

Research experiment on impact of foliar applied plant growth regulators on growth parameters and yield of hybrid rice (PHB-71) was conducted during Kharif season 2015 and 2016, in Randomized Block Design with three replications at Student Instructional Farm at C.S. Azad University of Agriculture and Technology Kanpur, The experiment carried out with foliar application of five growth regulators each in two concentration, total eleven treatment as $\mathrm{GA}_{3} 20$ and 40 ppm, IAA 25 and 50 ppm, NAA 10 and 20 ppm, Triacontanol 5 and 10 ppm, Cycocel 3000 and $4000 \mathrm{ppm}$ and control which were replicated three times, two foliar spray was done at tillering and at flowering time with sprayer. Nitrogen content in rice leaves was estimated by using instrument, semi- auto nitrogen analyzer of model KEL PLUSH. These instrument works on the principal of kjeldahl method of nitrogen analysis. Different observations were recorded as Total dry biomass of plant $(\mathrm{g})$, Total leaf area plant $^{-1}$, Total number of leaves plant $^{-1}$, at different stages. Days to $50 \%$ flowering, days to maturity, nitrogen content in straw (\%), Grain yield plant $^{-1}(\mathrm{~g})$.

\section{Results and Discussion}

The dry matter production is mainly due to higher translocation efficiency of assimilates source to sink, in pooled analysis over two year (Table 1) result indicated that maximum total dry biomass was recorded with the IAA $50 \mathrm{ppm}$ followed by TRIA $5 \mathrm{ppm}$ and others treatment of PGRs also give better response at $30,60,90 \mathrm{DAT}$ and at maturity stages of crop in both the years 2015 and 2016. These findings are in conformity to Aldesuquy H.S. (2001), Prajapati et al., (2017). Who obtained similar improvement in dry matter production by leaves and stem by the use of IAA and TRIA.

A morphological and important yield supporting character i.e. total leaf area plant $^{-1}$ showed in (Table 2) that the pooled analysis over two year data maximum found with IAA 50 followed by TRIA $5 \mathrm{ppm}$. Others plant growth regulators also enhance the total leaf area plant ${ }^{-1}$ in comparison to control in both the years 2015 and 2016. Finding was supported by Panday et al., (2001), Sutedja (1993).

In pooled analysis over two year data (Table 3) showed that total numbers of leaves was highly produced with the treatment of IAA 50 ppm as compare to TRIA $5 \mathrm{ppm}$ in different growth stages. Other growth hormones are also increases total number of leaves in rice crop in both years 2015 and 2016. Finding supported by Tripathi (1978), Prajapati et al., (2017).

Days to flowering and maturity pooled analysis over two year data (Table 4) indicated that CCC 3000 and $4000 \mathrm{ppm}$ give late flowering and maturity of the crop in both the year 2015 and 2016 strongly supported by Mehrotra et al., (1983), Dighe et al., (1985), Sexana et al., (1988). IAA and other treatment also delayed flowering and maturity with the observation of Pandit et al., (1986), Rajendra and Jones Jonathan (2009). Treatment of GA 3 caused early flowering supported by Naeem et al., (2004), Awan et al., (1999) and Iilium Lee et al., (1999) in both the year in rice crop. 
In pooled analysis over two year data showed that the maximum nitrogen content in straw at maturity stage showed (Table 4) was mainly affected by the foliar spray of IAA $50 \mathrm{ppm}$ followed by lower dose of IAA $25 \mathrm{ppm}$. Other hormones in different concentrations also accelerating the nitrogen value in straw. This result is favor by the discussion given by Patil et al., (1989), Prajapati et al., (2017).
Plant hormones increased the grain yield plant $^{-1}(\mathrm{~g})$ in pooled analysis over two year data (Table 4) indicate the highly produced by the foliar spray of IAA $50 \mathrm{ppm}$ followed by TRIA $5 \mathrm{ppm}$, others treatments in different concentration also effective in producing grain yield plant $^{-1}$. This conclusion was favored by Hussen (2011), Borowaski et al., (2000), Choi et al., (2010).

Table.1 Impact of various growth regulators on Total dry biomass of Hybrid Rice at different crop growth Stages

\begin{tabular}{|c|c|c|c|c|c|c|c|c|c|c|c|c|}
\hline \multirow{3}{*}{ Treatments } & \multicolumn{12}{|c|}{ Total dry biomass (g) } \\
\hline & \multicolumn{3}{|c|}{30 DAT } & \multicolumn{3}{|c|}{60 DAT } & \multicolumn{3}{|c|}{90 DAT } & \multicolumn{3}{|c|}{ Maturity } \\
\hline & 2015 & 2016 & Pooled & 2015 & 2016 & Pooled & 2015 & 2016 & Pooled & 2015 & 2016 & Pooled \\
\hline $\mathbf{T}_{1^{-}}$Control & 3.10 & 3.38 & 3.24 & 10.50 & 11.07 & 10.79 & 23.40 & 23.87 & 23.64 & 36.26 & 36.70 & 36.48 \\
\hline $\begin{array}{c}\mathbf{T}_{2}-\mathbf{G A}_{3} \mathbf{2 0} \\
\mathbf{p p m}\end{array}$ & 3.88 & 4.31 & 410 & 14.48 & 15.03 & 14.76 & 29.30 & 29.76 & 9.53 & 52.10 & 52.59 & 52.35 \\
\hline $\begin{array}{c}\mathbf{T}_{3}-\mathbf{G A}_{3} \mathbf{4 0} \\
\mathbf{p p m}\end{array}$ & 4.15 & 4. & 4.35 & 15.60 & 16.12 & 15.86 & 30.27 & 30.79 & 30.53 & 54.78 & 5.13 & 54.96 \\
\hline $\begin{array}{c}\text { T}_{4-} \text { IAA } 25 \\
\text { ppm }\end{array}$ & 4.12 & 4.53 & 4 & 14.70 & 15.25 & 14.98 & 31.40 & 31.95 & 31. & 56.55 & 56.91 & 56.73 \\
\hline $\begin{array}{c}\mathrm{T}_{5}-\text { IAA } 50 \\
\text { ppm }\end{array}$ & 4.56 & 5.1 & 4.79 & 16.41 & 1 & 16 & 10 & 51 & 33.36 & 70 & 2.06 & 38 \\
\hline $\begin{array}{c}\text { T. }_{6} \text { - TRIA } 5 \\
\text { ppm }\end{array}$ & 4.40 & 4 & & 60 & 5.15 & 15.88 & .80 & 30 & 32.05 & 9.00 & 9.36 & \\
\hline $\begin{array}{c}\text { T }_{7-} \text { TRIA } \\
10 \text { ppm }\end{array}$ & 4.00 & 4.28 & 14 & 4.80 & 15.33 & 15.07 & 27.93 & 28.57 & 28.25 & 53.34 & 53.80 & 53.57 \\
\hline $\begin{array}{c}\text { T8- NAA }_{8} \\
10 \text { ppm }\end{array}$ & 3.75 & 4.15 & 3 & 14.30 & 14.83 & 14.57 & 28.30 & 28.79 & 28.55 & 49.46 & 49.91 & 49.69 \\
\hline $\begin{array}{c}\text { T9-NAA } 20 \\
\text { ppm }\end{array}$ & 4.03 & 4.40 & 4 & 14.87 & 15.40 & 15.14 & 29.20 & 29.70 & 9.45 & 50.90 & 51.36 & 51.13 \\
\hline $\begin{array}{l}T_{10^{-}} \mathrm{CCC} \\
3000 \mathrm{ppm}\end{array}$ & 3.60 & 3.99 & 3.80 & 13.47 & 14.03 & 13.75 & 29.15 & 29.64 & 29.40 & 47.20 & 47.55 & 47.38 \\
\hline $\begin{array}{c}T_{11}-\mathrm{CCC} \\
4000 \mathrm{ppm}\end{array}$ & 3.35 & 3.75 & 3.55 & 12.28 & 12.82 & 12.55 & 26.62 & 27.14 & 26.88 & 43.70 & 44.18 & 43.94 \\
\hline $\operatorname{SE}(d)$ & 0.15 & 0.16 & 0.11 & 0.74 & 0.95 & 0.59 & 0.56 & 0.77 & 0.47 & 1.02 & 1.09 & 0.75 \\
\hline CD at $5 \%$ & 0.33 & 0.34 & 0.22 & 1.54 & 2.00 & 1.19 & 1.17 & 1.60 & 0.96 & 2.13 & 2.27 & 1.50 \\
\hline
\end{tabular}


Table.2 Impact of various growth regulators on Total leaf area plant ${ }^{-1}$ of Hybrid Rice at different growth Stages

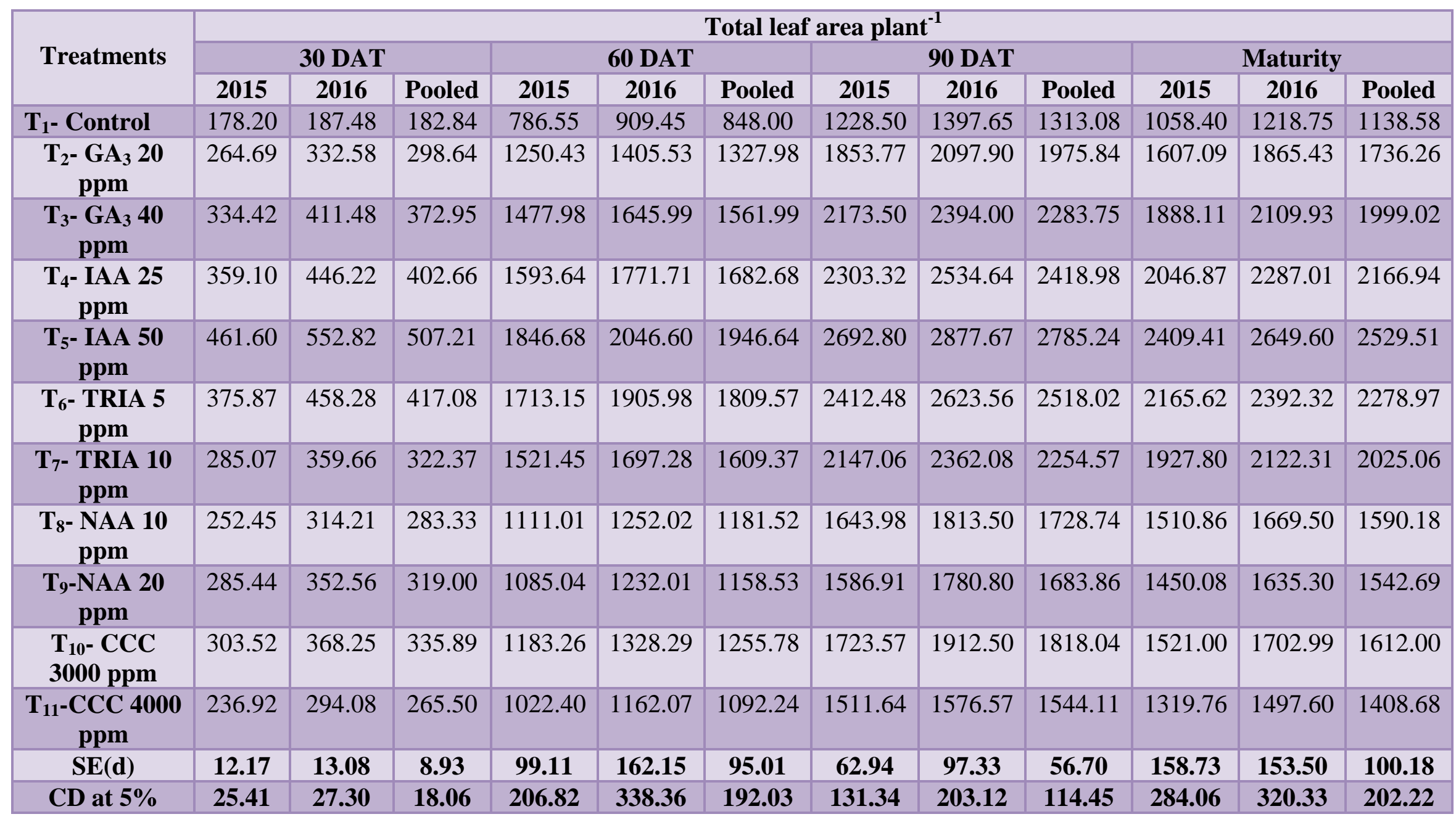


Table.3 Impact of various growth regulators on total number of leaves of hybrid rice at different growth Stages

\begin{tabular}{|c|c|c|c|c|c|c|c|c|c|c|c|c|}
\hline \multirow{3}{*}{ Treatments } & \multicolumn{12}{|c|}{ Total number of Leaves } \\
\hline & \multicolumn{3}{|c|}{30 DAT } & \multicolumn{3}{|c|}{60 DAT } & \multicolumn{3}{|c|}{90 DAT } & \multicolumn{2}{|c|}{ Maturity } & \multirow[b]{2}{*}{ Pooled } \\
\hline & 2015 & 2016 & Pooled & 2015 & 2016 & Pooled & 2015 & 2016 & Pooled & 2015 & 2016 & \\
\hline $\mathbf{T}_{1}-$ Control & 14.85 & 15.15 & 15.00 & 27.70 & 28.50 & 28.10 & 30.20 & 31.10 & 30.65 & 29.00 & 28.00 & 28.50 \\
\hline $\begin{array}{c}\mathbf{T}_{2}-\mathbf{G A}_{3} \mathbf{2 0} \\
\mathbf{p p m}\end{array}$ & 17.30 & 18.10 & 17.70 & 33.50 & 33.10 & 33.30 & 37.10 & 38.30 & 37.70 & 36.10 & 37.90 & 37.00 \\
\hline $\begin{array}{c}\mathbf{T}_{3}-\mathbf{G A}_{3} \mathbf{4 0} \\
\mathbf{p p m}\end{array}$ & 18.20 & 19.05 & 18.63 & 35.60 & 36.13 & 35.87 & 39.50 & 40.00 & 39.75 & 38.30 & 39.10 & 38.70 \\
\hline 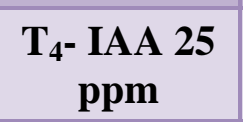 & 19.00 & 20.10 & 19.55 & 36.62 & 37.14 & 36.88 & 40.30 & 40.80 & 40.55 & 39.20 & 40.05 & 39.63 \\
\hline $\begin{array}{c}\text { T5- IAA } 50 \\
\text { ppm }\end{array}$ & 20.25 & 21.00 & 20.63 & 38.50 & 39.11 & 38.81 & 42.40 & 42.00 & 42.20 & 41.30 & 41.80 & 41.55 \\
\hline $\begin{array}{c}\text { T6- TRIA } 5 \\
\text { ppm }\end{array}$ & 19.35 & 20.10 & 19.73 & 37.40 & 38.09 & 37.75 & 40.90 & 41.10 & 41.00 & 40.00 & 40.60 & 40.30 \\
\hline $\begin{array}{c}\text { T }_{7-} \text { TRIA } \\
10 \mathrm{ppm}\end{array}$ & 18.10 & 19.03 & 18.57 & 36.50 & 37.10 & 36.80 & 39.70 & 40.15 & 39.93 & 39.00 & 39.30 & 39.15 \\
\hline $\begin{array}{l}\text { T}_{8} \text { - NAA } \\
10 \mathrm{ppm}\end{array}$ & 16.50 & 17.10 & 16.80 & 31.50 & 32.10 & 31.80 & 35.70 & 36.00 & 35.85 & 34.80 & 35.00 & 34.90 \\
\hline $\begin{array}{c}\text { T9-NAA } 20 \\
\text { ppm }\end{array}$ & 17.62 & 18.15 & 17.89 & 32.40 & 33.08 & 32.74 & 36.30 & 37.00 & 36.65 & 35.40 & 36.06 & 35.73 \\
\hline $\begin{array}{l}T_{10^{-}} \text {CCC } \\
3000 \text { ppm }\end{array}$ & 18.65 & 19.18 & 18.92 & 34.60 & 35.12 & 34.86 & 38.50 & 39.00 & 38.75 & 37.50 & 38.10 & 37.80 \\
\hline $\begin{array}{c}T_{11}-\mathrm{CCC} \\
4000 \mathrm{ppm}\end{array}$ & 17.55 & 18.07 & 17.81 & 33.40 & 34.07 & 33.74 & 37.30 & 38.00 & 37.65 & 36.20 & 37.00 & 36.60 \\
\hline SE(d) & 1.05 & 1.20 & 0.78 & 1.60 & 1.84 & 1.19 & 1.35 & 1.63 & 1.04 & 1.32 & 1.42 & 0.95 \\
\hline CD at $5 \%$ & 2.19 & 2.51 & 1.57 & 3.35 & 3.85 & 2.41 & 2.89 & 3.41 & 2.11 & 2.76 & 2.97 & 1.92 \\
\hline
\end{tabular}


Int.J.Curr.Microbiol.App.Sci (2019) 8(1): 838-845

Table.4 Impact of various growth regulators on $50 \%$ flowering and maturity, Nitrogen content in straw (\%) and, grain yield plant ${ }^{-1}(\mathrm{~g})$ of Hybrid Rice

\begin{tabular}{|c|c|c|c|c|c|c|c|c|c|c|c|c|}
\hline \multirow[t]{2}{*}{ Treatments } & \multicolumn{3}{|c|}{ Days to $50 \%$ flowering } & \multicolumn{3}{|c|}{ Days to maturity } & \multicolumn{3}{|c|}{$\begin{array}{l}\text { Nitrogen content in } \\
\text { straw }(\%)\end{array}$} & \multicolumn{3}{|c|}{ Grain yield plant $^{-1}(\mathrm{~g})$} \\
\hline & 2015 & 2016 & Pooled & 2015 & 2016 & Pooled & 2015 & 2016 & Pooled & 2015 & 2016 & Pooled \\
\hline $\begin{array}{c}T_{1^{-}} \\
\text {Control }\end{array}$ & 84.00 & 85.00 & 84.00 & 130.00 & 131.00 & 130.50 & 0.52 & 0.53 & 0.53 & 20.10 & 20.15 & 20.13 \\
\hline $\begin{array}{l}\mathrm{T}_{2-} \mathrm{GA}_{3} \\
20 \mathrm{ppm}\end{array}$ & 84.88 & 85.11 & 84.88 & 131.33 & 131.44 & 131.39 & 0.58 & 0.59 & 0.59 & 21.45 & 21.50 & 21.48 \\
\hline $\begin{array}{l}T_{3}-G_{3} \\
40 \mathrm{ppm}\end{array}$ & 84.99 & 86.00 & 84.99 & 131.77 & 131.88 & 131.83 & 0.59 & 0.60 & 0.60 & 22.65 & 22.70 & 22.68 \\
\hline $\begin{array}{l}T_{4}-\text { IAA } \\
25 \mathrm{ppm}\end{array}$ & 86.11 & 86.44 & 86.11 & 134.88 & 135.00 & 134.94 & 0.61 & 0.62 & 0.62 & 22.85 & 22.95 & 22.90 \\
\hline $\begin{array}{l}\text { T5 }_{5} \text { IAA } \\
50 \mathrm{ppm}\end{array}$ & 86.22 & 86.55 & 8 & 135.00 & 135.22 & 135.11 & 0.62 & 0.63 & 0.63 & 25.20 & 5.30 & 25.25 \\
\hline $\begin{array}{c}\text { T6- TRIA } \\
5 \mathrm{ppm}\end{array}$ & 87.33 & 87.77 & 3 & 133.66 & 77 & 133.72 & 0.60 & 0.61 & 0.61 & 4.26 & 4.30 & 24.28 \\
\hline $\begin{array}{c}\text { T }_{7} \text { - TRIA } \\
10 \mathrm{ppm}\end{array}$ & 87.11 & 87.33 & 87.11 & 133.44 & 133.66 & 133.55 & 0.59 & 0.60 & 0.60 & 23.00 & 23.10 & 23.05 \\
\hline $\begin{array}{l}T_{8^{-}} \text {NAA } \\
10 \mathrm{ppm}\end{array}$ & 85.44 & 85.66 & 85.44 & 132.22 & 132.44 & 132.33 & 0.56 & 0.57 & 0.57 & 22.85 & 22.90 & 22.88 \\
\hline $\begin{array}{l}\text { T9-NAA }_{9} \\
20 \mathrm{ppm}\end{array}$ & 85.66 & 85.88 & 85.66 & 132.44 & 132.66 & 132.55 & 0.57 & 0.58 & 0.58 & 21.70 & 21.80 & 21.75 \\
\hline $\begin{array}{l}T_{10^{-}} \mathrm{CCC} \\
3000 \mathrm{ppm}\end{array}$ & 87.44 & 87.99 & 87.44 & 135.11 & 135.33 & 135.22 & 0.56 & 0.57 & 0.57 & 22.60 & 22.65 & 22.63 \\
\hline $\begin{array}{c}T_{11}-\mathrm{CCC} \\
4000 \mathrm{ppm}\end{array}$ & 87.66 & 88.22 & 87.66 & 135.22 & 135.55 & 135.39 & 0.55 & 0.56 & 0.56 & 21.44 & 21.52 & 21.48 \\
\hline $\operatorname{SE}(d)$ & 0.88 & 0.87 & 0.60 & 1.06 & 1.39 & 0.86 & 0.011 & $\begin{array}{c}0.01 \\
4\end{array}$ & $\begin{array}{c}0.00 \\
9\end{array}$ & 1.02 & 1.04 & 0.71 \\
\hline CD at $5 \%$ & 1.84 & 1.82 & 1.22 & 2.21 & 2.91 & 1.73 & 0.025 & $\begin{array}{c}0.03 \\
0\end{array}$ & $\begin{array}{c}0.01 \\
9\end{array}$ & 2.14 & 2.18 & 1.44 \\
\hline
\end{tabular}

In conclusion foliar application of plant growth regulators (PGRs) mainly IAA was very effective for increasing dry biomass, total leaf area plant ${ }^{-1}$, total number of leaves, days to $50 \%$ flowering and maturity, nitrogen content in straw $(\%)$, grain yield plant ${ }^{-1}(\mathrm{~g})$ during both kharif years 2015 and 2016. The treatments of hormones most effective for physiological, morphological and yield parameters in hybrid rice crop.

\section{Acknowledgement}

The author are thankful for financial assistance by University Grant Commission, Gov. of India, New Delhi under Rajiv Gandhi National Fellowship (RGNF-2015-17-SCUTT-5033), and greatful to my advisory chairman Dr. S.P. Kushwaha for better guidance and supporting during my research work. 


\section{References}

Aldesuquy, H.S., 2001. Efficiency of indole3- acetic acid on improvement of some biochemical and physiological aspects of Wheat flag leaf during grain filling. Agrochimicia, 45(1/2); 1-13.

Awan, I.U., Baloch. M.S., Sadozai, N.S. and Sulemani, M.Z. 1999 stimulatory effect of $\mathrm{GA}_{3}$ and IAA on ripening process Kernel development and quality of rice Pakistan Journal Biological Science; 2 (2): 410-412.

Borowaski, E., Blamowaski, Z.K., Michalek, W. 2000 Effect of tomatoex/ triacontanol on chorophyll fluorescence and tomato (Lycopersicum esculentum Mill.) yield, Acta Physiologiae Plantarum 22, 271-274.

Borowski, E. and Blamowski, Z.K., 2009. The effects of triacontanol 'TRIA' and Asahi SL on the development and metabolic activity of sweet basil (Ocimum basilicum L.) plants treated with chilling. Folia Horticulturae Ann. 21(1): 39-48.

Choi, J. H., Abe, N., Tanaka, H., Fushimi, K., Nishina, Y., Morita, A., Kiriiwa, Y., Motohashi, R., Hashizume, D., Koshino, H. and Kawagishi, H. 2010. Plant-growth regulator, imidazole-4carboxamide, produced by the fairy ring formingfungus Lepista sordida. $J$. Agric Food Chem., 58(18): 99569959.

DAC\&FW (Annual Report 2016-17) Pp. 1-2

Davies, P.J. 1995. The plant hormones: Their nature, occurrence, and functions. In P.J. Davies (ed.) Plant hormones: Physiology, biochemistry and molecular biology. Kluwer Academic, Dordrecht, Boston, London.

Dighe, R.S., Thakre, J.T. and Lal, S.S. 1985. Panjabrao Krishi Vidhyapeeth Research Journal: 7(2): 12-15.
Hussein, M. M. and Alwa, A. K. 2014. Effect of zinc and absorbic acid application pigments of millet plants grown under different salinity. Agricultural sciences, 5:1253-1260.

Mehrotra, O.N. 1983. Effect of growth regulators on wheat Indian J. Agric. Res. 17 (1/2):57-61.

Naeem, M., Bhatti. I., Ahamad, R.H. and Ashraf, M.Y. 2004 Effect of low temperature Stress in transplanted Aman Rice varieties Mediated by Different Transplantig dates, Acadmic Journal of plant science 2 (3): 132138.

Pandey, N., Upadhyay, S. K., Tripathi, R. S. 2001. Effect of plant growth regulators and fertility levels on growth and yield of transplanted rice. Indian Journal of Agricultural Research; 35(3): 205207. 7 ref.

Patil, B. N. and Dhomne, M. G., 1998. Growth analysis and yied of sunflower as influenced by growth retardant. Annl. Physio., 12 (1): 29-31.

Prajapati, L., Kushwaha, S.P. and Singh, B. A. 2017 Physiological assessment of growth regulators on growth, yield and quality traits of basmati rice (Oryza sativa L.) Journal of Pharmacognosy and Phytochemistry; 6(5): 164-166

Rajendra, B., Jones Jonathan D.G. 2009 Role of plant hormones in plant defense responses. Plant Molecular Biology, 69:473-488.

Saxena, H. K., Tripathi, R.S. and Singh, O.P. 1988. Effect of growth regulators on crop efficiency and productivity of gram (Cicer arietinum L.). Paper presented at National Seminar on Recent Advanced in plant growth regulator research no. 17-19, 1988, Jodhpur.

Sutedja, I. N. 1993. Growth of vanilla short 
stem cuttings promoted by rootone $\mathrm{F}$ and Dharmasri 5 EC (in Indonesian). Udayana University Scientific Journal. 20 (38): 138 - 145.
U.S.D.A. Foreign Agriculture Service 2018. World Agriculture Production. Pp. 14.

\section{How to cite this article:}

Pawan Kumar Goutam, S.P. Kushwaha, Dharmadew Chauhan, Vikas Yadav and Subhash Kumar. 2019. Impact of Plant Growth Regulators on Growth, Phenology and yield Characters of Hybrid Rice. Int.J.Curr.Microbiol.App.Sci. 8(01): 838-845. doi: https://doi.org/10.20546/ijcmas.2019.801.091 